\title{
A Randomized Exploratory Study to Investigate the Inflammatory Response During an Ultraviolet- Radiation-Induced Cold Sore Episode
}

\author{
Joseph Veltri (D) - Ron Boon · Arne Böhling · Klaus-Peter Wilhelm • \\ Stephan Bielfeldt
}

Received: January 29, 2021 / Accepted: April 9, 2021 / Published online: April 28, 2021

(c) The Author(s) 2021

\begin{abstract}
Introduction: Antivirals and occlusive lip patches are key treatments for cold sores. Additional therapeutic options, and validated methods to evaluate these, are needed.

Methods: This exploratory, double-blind, single-center study aimed to evaluate a novel lip patch containing the antiviral aciclovir (ACV) using noninvasive methods for measuring cold sore-associated inflammation. Healthy subjects with ultraviolet radiation (UVR)-induced cold sores were randomized to 10 days' treatment with a lip patch containing ACV $(N=12)$ or without ACV $(N=13)$. Outcome measures included blood flux (field laser perfusion imaging, FLPI), skin temperature (thermography), and skin redness (high-resolution color photography, HRCP).
\end{abstract}

Supplementary Information The online version contains supplementary material available at https:// doi.org/10.1007/s13555-021-00531-X.

J. Veltri $(\square)$

GSK Consumer Healthcare, Medical Affairs Lip

Health, Parsippany, NJ, USA

e-mail: veltrijc1@gmail.com

R. Boon

GSK Consumer Healthcare, Medical Affairs Lip

Health, Brentford, Middlesex, UK

A. Böhling · K.-P. Wilhelm · S. Bielfeldt proDERM Institute for Applied Dermatological

Research, Schenefeld, Hamburg, Germany
Results: Mean blood flux (in perfusion units) and skin temperature (in ${ }^{\circ} \mathrm{C} /$ pixel) were higher for cold sores versus intrasubject control regions. For ACV versus placebo patches, skin temperature was higher for ACV with total day 1-5 mean values of 2.6 versus 0.5 $(p=0.036)$ and day 1-10 mean values of 3.2 versus $0.8(p=0.049)$. Conversely, mean total episode blood flux values over days 1-5 were lower for ACV versus placebo patch (flux 2227 versus 2939, $p=0.340$ ) and remained lower over days 1-10 (flux ACV 810 versus placebo $961, p=0.404)$. HRCP failed to discriminate cold sores from control regions or between treatments. Subject-reported pain/soreness, itching, and burning were generally lower with ACV patch.

Conclusions: FLPI reliably measures cold sorerelated inflammation and thermography heat radiating from the skin. HRCP was of little value.

Trial Registration: NCT01653509.

Keywords: Aciclovir; Cold sore; Inflammation; Lip patch; Ultraviolet radiation (UVR) 


\section{Key Summary Points}

\section{Why carry out this study?}

None of the current topical cold sore treatments provide a combination of an antiviral such as aciclovir incorporated into an occlusive lip patch.

This exploratory study aimed to evaluate and validate novel noninvasive methods for measuring cold-sore-associated inflammation suitable for antiviral lip patch research.

New outcome measures included field laser perfusion imaging (FLPI) to assess blood flux, thermography for skin temperature changes, and high-resolution color photography (HRCP) for skin redness.

\section{Study outcomes}

HRCP failed to discriminate cold sores from control regions, or between treatments.

Mean blood flux and skin temperature were higher for cold sores versus intrasubject control regions.

For aciclovir versus placebo patches, skin temperature was significantly higher and decrease in blood flux was numerically greater.

Preliminary data suggest an occlusive lip patch containing aciclovir is effective and well tolerated.

\section{Study learnings}

FLPI proved the most reliable measure of cold-sore-related inflammation and is worthy of further investigation in future lip patch and nonpatch cold sore studies.

\section{DIGITAL FEATURES}

This article is published with digital features, including summary slide, to facilitate understanding of the article. To view digital features for this article go to https://doi.org/10.6084/ m9.figshare.14388431.

\section{INTRODUCTION}

Recurrent herpes labialis (RHL, cold sore) is a common infection of the skin and mucus membranes predominantly caused by herpes simplex virus-1 (HSV-1) [1]. The worldwide seroprevalence of HSV-1 is estimated at $67 \%$, and it continues to be a lifelong physical and psychological burden for RHL sufferers [2-4]. More than 40 years after its discovery, the antiviral aciclovir (ACV) and its related compounds continue to be the mainstay of treatment for HSV infections [5, 6], including RHL. Antivirals can be administered orally or topically and are most effective against RHL when applied early, i.e., when a burning, itching, or tingling sensation occurs in the skin before the sore breaks out (prodrome) [7]. Randomized, controlled, multicenter trials have demonstrated that ACV 5\% cream significantly reduces time to healing and duration of lesion pain versus vehicle control in healthy adults with RHL [8-10].

An alternative to topical creams and ointments is the use of an occlusive, absorbent dressing designed not to adhere to the wound, which has been shown to provide efficient healing as well as symptomatic relief and discreet protection of an RHL lesion [7, 11]. There remains an unmet need for additional therapeutic options for RHL, such as a single product that combines the dual benefits of a cold sore patch to aid wound healing and an antiviral agent to treat the virus, as well as new alternative noninvasive techniques for measuring outcomes.

There are several challenges when conducting RHL clinical studies, such as the rapid onset of cold sore episodes (usually within $24 \mathrm{~h}$ from first sign or symptom), which affords only a narrow window for therapeutic intervention 
[12]. The infrequent occurrence of cold sores also necessitates large, costly studies. This has placed greater importance on the use of methods for cold sore induction in the early phases of clinical development to enable more effective screening of potential therapeutic candidates. There are different approaches to induction of cold sores in clinical studies, and considerable evidence, dating back to 1985 [13], supports the use of ultraviolet radiation (UVR) to induce RHL [14]. Whereas a specified standard erythema dose (SED) of UVR is now preferred for cold sore induction [15], local authorities often require that the minimal erythema dose (MED) of UVR be used. There are also methodological challenges associated with assessing cold sore progression underneath a patch.

A randomized, double-blind, placebo-controlled, single-center exploratory study was performed using UVR, based on MED and SED, to induce a cold sore in susceptible volunteers. The study aimed to evaluate the adequacy of noninvasive methods for measuring cold sore inflammation, provide preliminary descriptive data on the impact (efficacy, safety, and subjectreported experience) of adding ACV to an occlusive lip patch (versus placebo/reference lip patch), and inform the methodology and design of future studies. Three different methods previously validated for assessing inflammatory skins reactions were examined: full-field laser perfusion imaging (FLPI), thermography, and high-resolution color photography (HRCP) [16-20].

\section{METHODS}

Full details for all subsections of the methods are provided in the Electronic Supplementary Material. The study was approved by the German Federal Institute for Drugs and Medical Devices (Bundesinstitut für Arzneimittel und Medizinprodukte, BfArM) and was conducted in August and September 2012 at proDERM (Hamburg, Germany) in accordance with the Declaration of Helsinki, ISO 14155, national regulatory requirements, and the principles of Good Clinical Practice (ICH 1996). All subjects provided written informed consent.

\section{Study Design and Conduct}

This was an exploratory, phase I study (NCT01653509) conducted in two stages. First, the effectiveness of UVR exposure to induce cold sores was evaluated using MED converted to SED. The second stage evaluated the ability of three noninvasive techniques-FLPI, thermography, and HRCP-to discriminate inflammation between a cold sore/no cold sore and treatment/no treatment.

\section{Subjects}

Subjects were healthy adults aged $\geq 18$ years with self-reported history of $\geq 3$ RHL episodes within the previous 12 months that they associated with exposure to sunlight. All subjects had lightly pigmented skin, with a measured individual typography angle $>10$ to $\leq 55$, approximating to skin phototypes II-IV according to the Fitzpatrick scale [21]. Principal exclusion criteria are provided in the Electronic Supplementary Material.

\section{Objectives}

The overall purpose of the study is to evaluate the potential of new noninvasive techniques for evaluation and screening of potential treatments for RHL. The primary objective is to investigate the difference in inflammatory response during a UVR-induced cold sore episode between two occlusive lip patches, one with ACV and one without, assessed using noninvasive FLPI, thermography, and HRCP. The secondary objectives are to evaluate subject-reported experiences and the safety/tolerability of the patches, and determine the feasibility of using these new techniques to conduct future clinical studies to evaluate the effectiveness of ACV patches as a treatment for cold sores.

\section{UVR Exposure}

First, the sensitivity of each subject to UVR was determined by measuring MED. Within 7 days 
thereafter, subjects underwent cold sore induction on a single quadrant of the lips by exposure to four MEDs of UVR. The amount of UVR estimated by MED was also converted to SEDs, with the maximum permitted UVR dose set at 20 SEDs as a safety measure.

\section{Interventions}

Subjects were randomized 1:1 to lip patches with ACV (the intervention) or lip patches without ACV (placebo/reference), both provided by the study sponsor [GlaxoSmithKline (GSK) Consumer Healthcare]. Randomization was according to a schedule prepared by the biostatistics department of the sponsor. Trained clinical staff applied the first lip patch and instructed subjects in correct application technique. Subjects applied one patch to the cold sore (maximum five patches $/ 24 \mathrm{~h}$ ) to ensure complete and continuous protection. Treatment lasted $\leq 10$ days, or until the cold sore healed. Examiners and subjects were blinded to treatment.

\section{Assessments}

Each subject served as their own control. The cold sore and a defined control region on the contralateral side of the lip were evaluated in the clinic on days $1,2,3,4,5,7$, and 10 using FLPI, thermography, and HRCP. Each method is described below and described in more detail in the Electronic Supplementary Material. Cold sore measurements were conducted with the patch in place, while the control region remained uncovered. For each method, the whole mouth was assessed, and the cold sore/control region defined by a single trained technician.

FLPI was performed using a Moor FLPI instrument (Moor Instruments Ltd., Axminster, UK). Back scattering of light waves following laser light illumination of the skin results in a speckle pattern that is dependent on capillary blood cell movement within $1 \mathrm{~mm}$ beneath the skin surface. The change of local contrast within the speckle pattern-blood flux-is the average speed multiplied by the concentration of blood cells, reported in perfusion units $[22,23]$.

A VarioCAM 480/25 thermography camera (Infratech $\mathrm{GmbH}$, Dresden, Germany) with a resolution of $\pm 0.035 \mathrm{~K}$ was used to measure heat radiating from the skin surface. Results are reported in ${ }^{\circ} \mathrm{C} /$ pixel of area scanned.

A high-resolution camera (model H4D31; Hasselblad, Gothenburg, Sweden) with 31-megapixel physical resolution, 16-bit color resolution, and standard flash system was used to quantify skin redness as an indicator of inflammation. A color standard was used, and raw images were converted to color values in the $L^{*}-a^{*}-b^{*}$ and $Y-x-y$ spaces.

\section{Subject-Reported Outcomes}

Each subject completed a daily study diary and answered a series of 12 questions on patch comfort, symptom intensity, and cold sore noticeability. Answers were given on a fivepoint categorical scale, ranging from " $1=$ Never bothered" to " $5=$ Bothered all the time," or " 1 = Strongly disagree" to " $5=$ Completely agree" (Supplementary Table S1).

\section{Safety Assessments}

Safety and tolerability were assessed by the number and type of adverse events (AEs), treatment-emergent AEs, and serious AEs (see the Electronic Supplementary Material for definitions). All AEs were assessed from the start of UVR exposure until study completion.

\section{Data and Statistical Analysis}

Sample size for this exploratory study was driven pragmatically with an aim of including 24 randomized subjects providing $\geq 20$ evaluable subjects (10 per treatment group). FLPI, thermography, and HRCP measurements taken at the site of the cold sore and control region were used to calculate the daily difference between the two sites. These differences were summed to calculate the total episode value for the 10-day treatment period; the "maximum episode value" was the maximum observed daily difference. Treatment effectiveness was evaluated by testing $\mathrm{H}_{0}$ : no difference between patches with 
Table 1 Baseline characteristics and UVR exposure for the study subjects

\begin{tabular}{|c|c|c|c|}
\hline Characteristic & $\mathrm{ACV}$ patch $(N=12)$ & Placebo patch $(N=13)$ & $\begin{array}{l}\text { Overall } \\
(N=25)\end{array}$ \\
\hline Female, $n(\%)$ & $11(91.7)$ & $12(92.3)$ & $23(92.0)$ \\
\hline Caucasian, $n(\%)$ & $12(100.0)$ & $13(100.0)$ & $25(100.0)$ \\
\hline Age, years, median (range) & $48(24-79)$ & $40(22-69)$ & $43(22-79)$ \\
\hline \multicolumn{4}{|l|}{ ITA skin type, $n(\%)^{\mathrm{a}, \mathrm{b}}$} \\
\hline$>41.0-55.0$ & $7(58.3)$ & $7(53.8)$ & $14(56.0)$ \\
\hline$>28.0-41.0$ & $5(41.7)$ & $5(38.5)$ & $10(40.0)$ \\
\hline$>10.0-28.0$ & $0(0)$ & $0(0)$ & $0(0)$ \\
\hline \multicolumn{4}{|c|}{ Actual UVR exposure, median (range) ${ }^{2}$} \\
\hline Minutes & $10.2(6.0-14.0)$ & $12.1(5.0-14.0)$ & $11.0(5.0-14.0)$ \\
\hline SEDs & $16.9(10.0-20.0)$ & $17.8(9.0-20.0)$ & $17.2(9.0-20.0)$ \\
\hline \multicolumn{4}{|c|}{ Actual versus planned UVR exposure, $n(\%)^{2}$} \\
\hline$>95 \%$ & $8(66.7)$ & $8(61.5)$ & $16(64.0)$ \\
\hline $80-95 \%$ & $2(16.7)$ & $3(23.1)$ & $5(20.0)$ \\
\hline$<80 \%$ & $2(16.7)$ & $1(7.7)$ & $3(12.0)$ \\
\hline
\end{tabular}

$A C V$ aciclovir, ITA individual typography angle, SED standard erythema dose, UVR ultraviolet radiation

${ }^{a}$ Where $>41.0-55.0,>28.0-41.0$, and $>10.0-28.0$ approximate to Fitzpatrick phototypes II, III, and IV, respectively [21]

b Data missing for $N=1$ (placebo patch group)

ACV and patches without ACV, versus $\mathrm{H}_{1}$ : a difference between the patches. Total episode value and maximum episode value were compared between treatment groups by calculating 95\% confidence intervals and using analysis of variance. Subject-reported outcomes were examined using descriptive statistics. Additional exploratory analyses are listed in the Electronic Supplementary Material.

\section{RESULTS}

\section{Subject Disposition and Demographics}

The study was conducted between July and September 2012. A total of 105 subjects were screened for possible inclusion in the study, of whom 67 (63.8\%) underwent initial MED determination (UVR exposure to the inner forearm). It was anticipated that $40 \%$ of subjects were likely to develop a cold sore [9]; therefore 60 of the 67 subjects received UVR on the lip. In total, 25 subjects were randomized to the ACV lip patch $(N=12)$ or placebo/reference lip patch $(N=13)$. These subjects composed the intention-to-treat population. Baseline characteristics are presented in Table 1 . Some data were missing for one subject; this subject was excluded in the per-protocol analyses, the data for which were consistent with the intention-totreat analyses and are not reported here. All randomized subjects completed the study.

\section{Irradiation Device}

The UV source used was based on a UVB (broad band) DermaPalt handheld home phototherapy device (Daavlin, Bryan, OH, USA) with FS type PLS9W/12 lamp (Philips, Poland). The device 

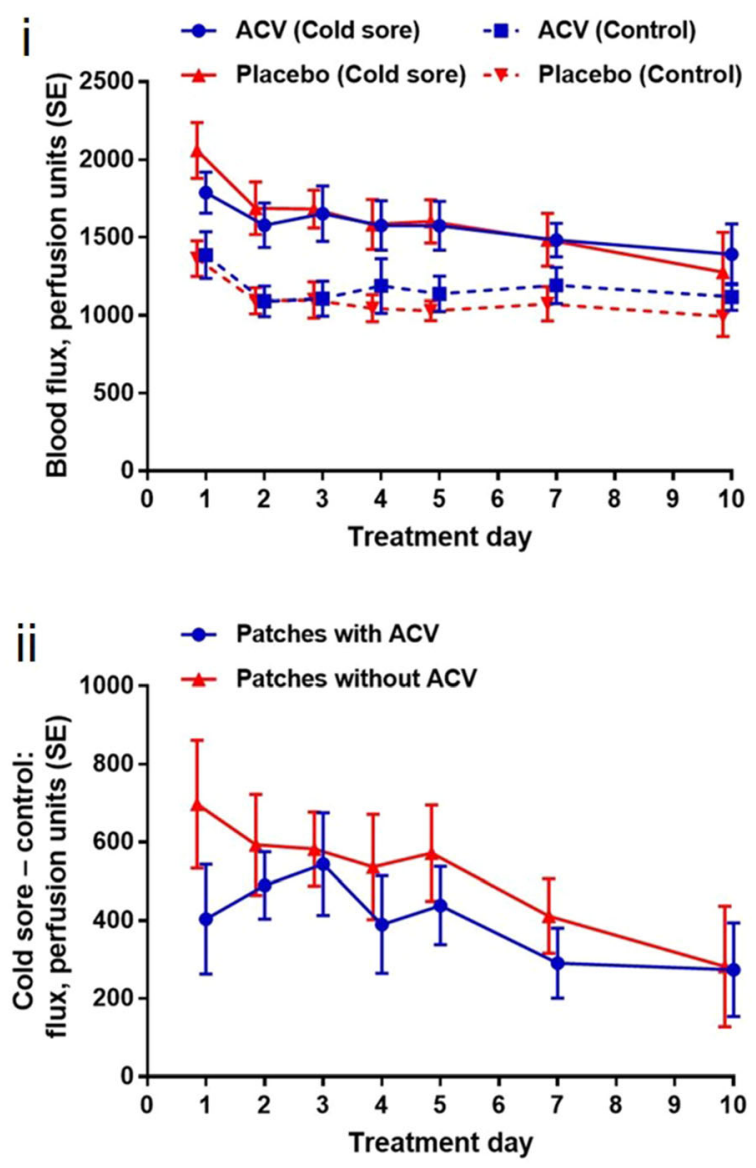

Fig. 1 Blood flux measured by FLPI. (i) FLPI measurement of mean daily blood flux for cold sore and control regions in subjects with UVR-induced cold sores randomized to treatment with ACV lip patches $(N=12)$ or placebo/reference patches $(N=13)$. (ii) Difference (cold sore - control region) in mean daily blood flux measured by FLPI for the ACV lip patch $(N=12)$ and placebo/ reference lip patch $(N=13)$ treatment groups. $A C V$ aciclovir, FLPI field laser perfusion imaging, $S E$ standard error, $U V R$ ultraviolet radiation

was fit with an adapter to hold a disposable 16-oz Styrofoam cup, with the bottom partly removed, mounted over the lamp wand [14]. The bottom hole of the cup was applied with light pressure to the test area on the lip for irradiation.

For MED determination, the small end of the Styrofoam cup was fit with a filter wheel containing five 12.5-mm-diameter UV-neutral density filters with optical density of $0.5,0.4$, $0.3,0.2$, and 0.1 and one unfiltered opening.
This filter wheel allows a series of six different UV exposures, increasing in intensity by $25 \%$ increments, to be simultaneously delivered to the subject's forearm in a single timed exposure.

The device was mounted to a stand to ensure easy handling and stable positioning when irradiating the skin or lip. It was cooled by air with an external ventilator.

\section{UVR Exposure and Clinical Characteristics}

UVR exposure ranged from 5 to 14 min (mean $10.8 \mathrm{~min}$ ), equivalent to 9-20 SEDs (mean 16.4 SEDs) (Table 1). As UVR exposure was capped at a maximum of 20 SEDs for safety reasons, some subjects did not receive the full planned UVR dose of four MEDs (Table 1). Of the 60 subjects who underwent UVR exposure on the lip, a greater proportion with skin type III/IV (10/17, $58.8 \%$ ) developed a UVR-induced cold sore than did subjects with skin type II $(14 / 43,32.6 \%)$.

The median time from UVR exposure to initial cold sore symptoms was $31.1 \mathrm{~h}$ in the 25 subjects included in the study. The median (range) time from first symptoms to clinic visit was $12.8(0.9-23.0) \mathrm{h}$. Subjects using the ACV patch had a shorter median healing time than subjects using the placebo/reference patch $(6.0$ versus 9.0 days), although the range was identical for both groups (3.0-9.0 days). Over the 10-day treatment period, the mean number of patches applied per day was approximately three; the lowest number was on day 10 (1.4 in each group), by which time half the subjects' cold sores had healed. For most time points, the mean number of patches used was slightly higher in the placebo group (overall mean: 3.1 placebo versus $2.8 \mathrm{ACV}$ ).

\section{Field Laser Perfusion Imaging}

Mean blood flux was greater in regions with a cold sore versus control regions (Fig. 1a). This was true for the ACV and placebo patch groups across the 10-day treatment period, suggesting that FLPI was able to discriminate a cold sore from a region without one. There was also a trend for a decreasing difference in blood flux across the treatment period for both groups 
Table 2 Total and maximum episode values for blood flux as measured by FLPI in subjects with UVR-induced cold sores

\begin{tabular}{|c|c|c|c|c|}
\hline & \multicolumn{2}{|l|}{ Adjusted mean (SE) } & \multirow[t]{2}{*}{ Mean difference $(95 \% \mathrm{CI})^{\mathrm{a}}$} & \multirow[t]{2}{*}{$p$-Value } \\
\hline & $\overline{\mathrm{ACV}}$ patch $(N=12)$ & Placebo patch $(N=13)$ & & \\
\hline \multicolumn{5}{|l|}{ Total episode value ${ }^{b}$} \\
\hline Days $1-5$ & $2227(526.5)$ & $2939(505.8)$ & $-712(-2222.5,798.1)$ & 0.340 \\
\hline Days $1-10$ & $2559(627.9)$ & $3470(603.2)$ & $-911(-2712.7,889.7)$ & 0.306 \\
\hline Maximum episode value ${ }^{c}$ & $810(127.9)$ & $961(122.9)$ & $-151(-518.0,216.0)$ & 0.404 \\
\hline
\end{tabular}

(Fig. 1b). The consistently smaller difference in the ACV patch group suggests that ACV may lead to reduced blood flow to a greater degree than placebo/reference. While numerically lower with the ACV patch, there were no significant differences in mean total episode value or mean maximum episode value between the groups (Table 2).

\section{Thermography}

A trend toward slightly higher mean skin temperature for cold sores compared with control regions was observed (Fig. 2a). This was evident across the 10-day treatment period and was more pronounced in the ACV patch group. The mean difference in temperature remained largely consistent for both treatment groups and was higher for the ACV patch group (Fig. 2b; Table 3). Consistent with this, mean total episode value was significantly higher with the ACV patch versus placebo/reference (days 1-10: mean difference $(95 \%$ CI) 2.5 (0.02, 4.93), $p=0.049$ ) (Table 3). Maximum episode value was also numerically higher for ACV, but this did not reach statistical significance (MEV: mean difference $(95 \% \mathrm{CI}) 0.4(-0.05,0.82)$, $p=0.080$ ) (Table 3).

\section{High-Resolution Color Photography}

HRCP, as a measure of skin redness, revealed generally higher mean skin color on the red-green ${ }^{\mathrm{a}^{*}}$ axis (i.e., redness), but lower mean skin color on the blue-yellow ${ }^{b^{*}}$ axis, for cold sores compared with control regions (Supplementary Fig. S1). This was apparent across the 10-day treatment period for both groups. Throughout the treatment period, mean differences in skin color on the $\mathrm{a}^{\mathrm{a}^{*}}$ and $b^{*}$ axes between cold sore regions and control regions were small and variable for both treatments (Supplementary Fig. S1). These data suggest that HRCP cannot discriminate between cold sore and control regions.

\section{Subject-Reported Outcomes}

Subject-reported scores for pain/soreness, itching, and burning were generally lower for the ACV patch compared with the placebo/reference patch. Subjects using the ACV patch reported less interference with facial movements and considered the patch less bothersome. All other subject-reported parameters were comparable between the ACV and reference patch. 

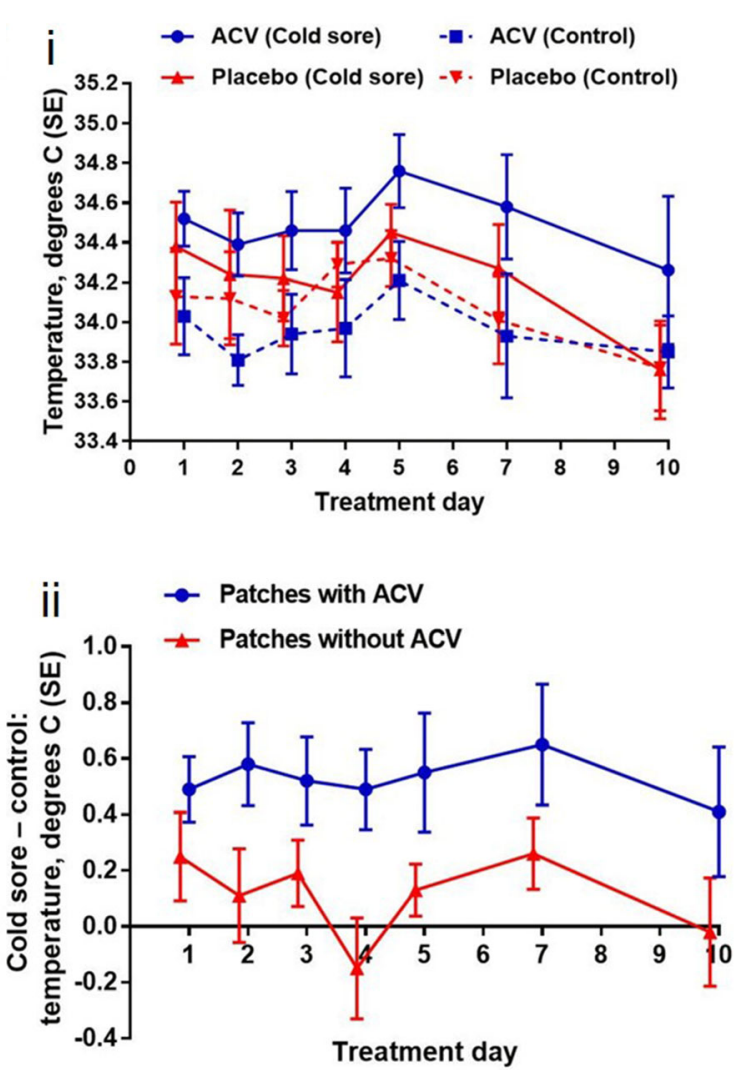

Fig. 2 Skin temperature measured by thermography. (i) Thermography measurement of mean daily skin temperature for cold sore and control regions in subjects with UVR-induced cold sores randomized to treatment with ACV lip patches $(N=12)$ or placebo/reference patches $(N=13)$. (ii) Difference (cold sore - control region) in mean daily skin temperature measured by thermography for the ACV lip patch $(N=12)$ and placebo/reference lip patch $(N=13)$ treatment groups. $A C V$ aciclovir, $S E$ standard error, UVR ultraviolet radiation

\section{Safety}

Three subjects experienced a total of three treatment-emergent AEs: one episode each of hyperkeratosis of the lip and lip erosion (both considered treatment related), and one episode of oral herpes. All were mild in intensity and occurred with the reference patch. There were no serious AEs.

\section{DISCUSSION}

Management of RHL episodes centers on the use of oral and topical agents, and the application of nonmedicated lip patches. Antivirals such as $\mathrm{ACV}$ and related compounds remain the mainstay therapeutic agents $[5,6]$. This randomized, placebo-controlled, exploratory study aimed to provide preliminary data on the suitability of three noninvasive methods for assessing coldsore-related inflammation. The study also provided early data on a novel treatment combining an occlusive lip patch with ACV.

The present study evaluated three methods for the assessment of cold-sore-associated inflammation. To the authors' knowledge, no previous studies have reported on the suitability of noninvasive methods for evaluating the inflammatory response as a measure of the effectiveness of lip patches during cold sore episodes. The methodologies were chosen as techniques for measuring three hallmark signs of inflammation (erythema, heat, and swelling) and based on their prior validation for the assessment of inflammatory skin reactions associated with trauma, irritants, and conditions such as acne and scleroderma [16-20]. This exploratory study now provides novel data regarding the ability of these noninvasive methods to measure inflammatory response and characterize treatment effect during cold sore episodes. While the performance of HRCP was inconsistent, and thermography provided limited discrimination and was potentially susceptible to uncontrolled external factors (e.g., fluctuation in a subject's skin temperature or room temperature), FLPI proved reliable and consistent, supporting its use in future studies. A point for further clarification, however, is the potential impact of the large difference in blood flux with the ACV patch, relative to placebo, that was seen within the first hour after patch application on day 1 .

Skin temperature determined by thermography was slightly higher (approximately $0.5^{\circ} \mathrm{C}$ ) at the site of active cold sores relative to control regions. This is consistent with cold-sore-associated inflammation, though physiological variability in skin temperature of up to $0.5^{\circ} \mathrm{C}$ 
Table 3 Total and maximum episode values for skin temperature as measured by thermography in subjects with UVRinduced cold sores

\begin{tabular}{|c|c|c|c|c|}
\hline & \multicolumn{2}{|l|}{ Adjusted mean (SE) } & \multirow[t]{2}{*}{ Mean difference $(95 \% \mathrm{CI})^{\mathrm{a}}$} & \multirow[t]{2}{*}{$p$-Value } \\
\hline & $\overline{\mathrm{ACV}}$ patch $(N=12)$ & Placebo patch $(N=13)$ & & \\
\hline \multicolumn{5}{|l|}{ Total episode value ${ }^{b}$} \\
\hline Days $1-5$ & $2.6(0.67)$ & $0.5(0.64)$ & $2.1(0.15,3.94)$ & 0.036 \\
\hline Days $1-10$ & $3.2(0.86)$ & $0.8(0.82)$ & $2.5(0.02,4.93)$ & 0.049 \\
\hline Maximum episode value ${ }^{c}$ & $0.9(0.15)$ & $0.5(0.15)$ & $0.4(-0.05,0.82)$ & 0.080 \\
\hline
\end{tabular}

has been reported in healthy adults [19]. However, the observation of a persistent trend for higher skin temperature with the ACV patch relative to the placebo patch was unexpected. This was opposite to the anticipated effect, particularly given the apparent ACV-associated decrease in inflammatory response determined by FLPI. Possible explanations include: thermography only measures surface temperature, whereas changes in blood flow measured by FLPI occur deeper within the dermis; a potential heat-buffering effect caused by greater edema fluid present at the site of cold sores treated with placebo patches; the process associated with more rapid healing with the ACV patch led to a temperature increase; and the possibility that ACV was associated with mild skin irritation that caused a detectable, though not clinically important, temperature increase. Further research is needed to learn whether this finding is reproducible, and to better understand the biological nature of the effect.

At the stipulation of the local authority, UVR-induced reactivation of cold sores in this study was performed based on the determination of MED. This is a subjective assessment of whether a particular degree of erythema occurs after exposure to a graded series of UVR doses, as interpreted by an evaluator [24, 25]. Although widely used, this method has limitations and a specified SED dose of UVR is now the preferred approach for UVR cold sore induction [15]. Therefore, in the present study, UVR exposure was converted to SEDs and a maximum permitted exposure was applied as a safety measure. Capping UVR exposure at 20 SEDs was a highly effective approach for UVR cold sore induction, with successful cold sore reactivation achieved in $40 \%$ of cases. This is consistent with previous studies using MED $[9,14,26]$. Furthermore, the good response rates observed using a maximum of 20 SEDs supports omission of the initial UVR sensitivity determination by MED from future studies and use of 20 SEDs on all subjects, thereby reducing UVR exposure and the burden placed on subjects. Only a high variability of UV sensitivity among subjects in a study would restrict the use of a fixed 20-SED irradiation. By excluding from the study subjects who receive UV therapy [27], take drugs that increase photosensitivity [28], or suffer from inflammatory diseases [29], the risk of overdosed UV irradiation can be minimized.

The study has several limitations. First, the sample size was small. In the absence of methods previously validated for the assessment of the inflammatory response associated with active cold sores, this exploratory study was performed to investigate the potential suitability of different noninvasive methods to measure cold-sore-associated inflammation. Second, as the study was exploratory, it was not designed or powered to detect statistically significant differences but rather to provide preliminary 
data to inform the design of a larger follow-up clinical study. Nevertheless, there were clear trends in the data and the key aim of evaluating different methodologies was met. Inclusion of ACV on lip patches appears to benefit individuals with cold sores by decreasing inflammation, as shown using FLPI by the trend toward a greater reduction in capillary blood flow versus placebo. The potential benefits of the ACV patch are further supported by observed reductions in healing time and number of patches required per cold sore episode, and by subjects' reporting ACV patches to interfere less with facial movements and be less bothersome. The clinical relevance of the small changes observed is unknown, as is the true benefit of ACV as an ancillary medicinal ingredient added to lip patches for treatment of cold sores. Such questions may be addressed in larger clinical studies that are appropriately powered to identify genuine differences in cold-sore-associated inflammation.

\section{CONCLUSIONS}

This exploratory study conducted in healthy adults with UVR-induced RHL successfully met its objectives of evaluating the adequacy of different methods for measuring cold sore inflammation and to provide preliminary data on the inclusion of the antiviral ACV in occlusive lip patches. From a practical and safety perspective, the use of up to 20 SEDs for UVR induction of cold sores represents an effective strategy that avoids the initial UVR sensitivity determination required using MED. FLPI is a useful and reliable tool for measuring and differentiating the inflammatory response associated with cold sores. Preliminary data indicate that FLPI is also suitable as a method to assess the potential efficacy of a combined cold sore treatment based on an occlusive patch containing ACV. These promising data warrant larger studies and support the use of FLPI to further evaluate the benefit of therapeutic lip patches in the treatment of cold sores.

\section{ACKNOWLEDGEMENTS}

The authors would like to thank the subjects who participated in this study.

Funding. This study and the journal's Rapid Services Fee were funded by GSK Consumer Healthcare (ClinicalTrials.gov ID: NCT01653509).

Authorship. All named authors meet the International Committee of Medical Journal Editors (ICMJE) criteria for authorship for this article, take responsibility for the integrity of the work as a whole, and have given their approval for this version to be published.

Authorship Contributions. Klaus-Peter Wilhelm was the principal Investigator and was involved in all activities from conceptualization of study design, supervision of execution, interpretation of results to review of manuscript. Arne Böhling was the study manager at the study site. Stephan Bielfeldt was technical advisor and contributed to study concept and protocol, study performance, data analysis and interpretation. Joseph Veltri and Ron Boon were responsible for the conception and design of the study. Jospeh Veltri wrote the study protocol and implemented the measurement techniques with assistance from Professor Wilhelm and his team at Proderm. Joseph Veltri and Ron Boon reviewed the data and Joseph Veltri wrote the draft of the study report. Ron Boon reviewed and edited the study report which formed the basis of the submission.

Medical Writing, Editorial, and Other Assistance. The authors are also grateful to Dr. Angela Hougardy (proDERM) for assistance with project management, Teresa Bacon (formerly of GSK Consumer Healthcare) for helpful discussions, and Darren Targett (formerly a statistical consultant to GSK Consumer Healthcare) for assistance with biostatistical analysis. Editorial support in the development of the manuscript was provided by Gardiner-Caldwell Communications, Macclesfield, UK, and was funded by GSK Consumer Healthcare. 
Disclosures. Joseph Veltri and Ron Boon were employees of GSK Consumer Healthcare at the time the research was conducted. Arne Böhling was an employee of proDERM Institute for Applied Dermatological Research at the time the research was conducted. Klaus-Peter Wilhelm and Stephan Bielfeldt are employees of proDERM Institute for Applied Dermatological Research.

Compliance with Ethics Guidelines. The study was approved by the German Federal Institute for Drugs and Medical Devices (Bundesinstitut für Arzneimittel und Medizinprodukte, BfArM) and was conducted in August and September 2012 at proDERM (Hamburg, Germany) in accordance with the Declaration of Helsinki, ISO 14155, national regulatory requirements, and the principles of Good Clinical Practice [ICH 1996]. All subjects provided written informed consent.

Data Availability. The dataset supporting the conclusions of this article is available from the ClinicalTrials.gov website (NCT01653509) and all data can be supplied upon request.

Open Access. This article is licensed under a Creative Commons Attribution-NonCommercial 4.0 International License, which permits any non-commercial use, sharing, adaptation, distribution and reproduction in any medium or format, as long as you give appropriate credit to the original author(s) and the source, provide a link to the Creative Commons licence, and indicate if changes were made. The images or other third party material in this article are included in the article's Creative Commons licence, unless indicated otherwise in a credit line to the material. If material is not included in the article's Creative Commons licence and your intended use is not permitted by statutory regulation or exceeds the permitted use, you will need to obtain permission directly from the copyright holder. To view a copy of this licence, visit http://creativecommons.org/licenses/by$\mathrm{nc} / 4.0 /$.

\section{REFERENCES}

1. Leplina O, Starostina N, Zheltova O, Ostanin A, Shevela E, Chernykh E. Dendritic cell-based vaccines in treating recurrent herpes labialis: results of pilot clinical study. Hum Vaccin Immunother. 2016;12(12):3029-35.

2. Looker KJ, Magaret AS, May MT, Turner KM, Vickerman P, Gottlieb SL, et al. Global and regional estimates of prevalent and incident herpes simplex virus type 1 infections in 2012. PLoS ONE. 2015;10(10):e0140765.

3. Spruance SL. The natural history of recurrent oralfacial herpes simplex virus infection. Semin Dermatol. 1992;11(3):200-6.

4. Fatahzadeh M, Schwartz RA. Human herpes simplex virus infections: epidemiology, pathogenesis, symptomatology, diagnosis, and management. J Am Acad Dermatol. 2007;57(5):737-63.

5. James SH, Prichard MN. Current and future therapies for herpes simplex virus infections: mechanism of action and drug resistance. Curr Opin Virol. $2014 ; 8: 54-61$.

6. Vere Hodge RA, Field HJ. Antiviral agents for herpes simplex virus. Adv Pharmacol. 2013;67:1-38.

7. Karlsmark T. Clinical study to evaluate performance of a colloid adhesive patch on recurrent herpes labialis lesions. Presented at the Annual Meeting of the Congress of the European Academy of Dermatology and Venerology. Rhodes, Greece; 2006.

8. Spruance SL, Nett R, Marbury T, Wolff R, Johnson J, Spaulding T. Acyclovir cream for treatment of herpes simplex labialis: results of two randomized, double-blind, vehicle-controlled, multicenter clinical trials. Antimicrob Agents Chemother. 2002;46(7):2238-43.

9. Evans TG, Bernstein DI, Raborn GW, Harmenberg J, Kowalski J, Spruance SL. Double-blind, randomized, placebo-controlled study of topical 5\% acyclovir$1 \%$ hydrocortisone cream (ME-609) for treatment of UV radiation-induced herpes labialis. Antimicrob Agents Chemother. 2002;46(6):1870-4.

10. Spruance SL, McKeough MB. Combination treatment with famciclovir and a topical corticosteroid gel versus famciclovir alone for experimental ultraviolet radiation-induced herpes simplex labialis: a pilot study. J Infect Dis. 2000;181(6):1906-10.

11. Karlsmark T, Goodman JJ, Drouault Y, Lufrano L, Pledger GW. Randomized clinical study comparing Compeed cold sore patch to acyclovir cream $5 \%$ in 
the treatment of herpes simplex labialis. J Eur Acad Dermatol Venereol. 2008;22(10):1184-92.

12. Harmenberg J, Oberg B, Spruance S. Prevention of ulcerative lesions by episodic treatment of recurrent herpes labialis: a literature review. Acta Derm Venereol. 2010;90(2):122-30.

13. Spruance SL. Pathogenesis of herpes simplex labialis: experimental induction of lesions with UV light. J Clin Microbiol. 1985;22(3):366-8.

14. Sayre RM, Dowdy JC, Harris KA, Berg JE, Trimble MW. A practical UV source to induce Herpes simplex labialis lesions in the clinic. Photodermatol Photoimmunol Photomed. 2007;23(1):20-3.

15. International Organization for Standardization (ISO) and Commission Internationale de l'Éclairage (CIE). Erythema reference action spectrum and standard erythema dose. International standard ISO 17166:1999(E) CIE S 007/E-1998. First edition 1999-12-15; corrected and reprinted 2000-11-15; 1999.

16. Do TT, Zarkhin S, Orringer JS, Nemeth S, Hamilton $\mathrm{T}$, Sachs D, et al. Computer-assisted alignment and tracking of acne lesions indicate that most inflammatory lesions arise from comedones and de novo. J Am Acad Dermatol. 2008;58(4):603-8.

17. Fullerton A, Rode B, Serup J. Skin irritation typing and grading based on laser Doppler perfusion imaging. Skin Res Technol. 2002;8(1):23-31.

18. George J, Bensafi A, Schmitt AM, Black D, Dahan S, Loche $\mathrm{F}$, et al. Validation of a non-contact technique for local skin temperature measurements. Skin Res Technol. 2008;14(4):381-4.

19. Howell KJ, Lavorato A, Visentin MT, Smith RE, Schaefer G, Jones CD, et al. Validation of a protocol for the assessment of skin temperature and blood flow in childhood localised scleroderma. Skin Res Technol. 2009;15(3):346-56.
20. Mattsson U, Jonsson A, Jontell M, Cassuto J. Digital image analysis (DIA) of colour changes in human skin exposed to standardized thermal injury and comparison with laser Doppler measurements. Comput Methods Programs Biomed. 1996;50(1): 31-42.

21. Fitzpatrick TB. The validity and practicality of sunreactive skin types I through VI. Arch Dermatol. 1988;124(6):869-71.

22. Briers JD. Laser Doppler, speckle and related techniques for blood perfusion mapping and imaging. Physiol Meas. 2001;22(4):R35-66.

23. Cheng H, Luo Q, Liu Q, Lu Q, Gong H, Zeng S. Laser speckle imaging of blood flow in microcirculation. Phys Med Biol. 2004;49(7):1347-57.

24. Farr PM, Diffey BL. Quantitative studies on cutaneous erythema induced by ultraviolet radiation. $\mathrm{Br}$ J Dermatol. 1984;111(6):673-82.

25. Farr PM, Diffey BL. The vascular response of human skin to ultraviolet radiation. Photochem Photobiol. 1986;44(4):501-7.

26. Boon R, Goodman JJ, Martinez J, Marks GL, Gamble M, Welch C. Penciclovir cream for the treatment of sunlight-induced herpes simplex labialis: a randomized, double-blind, placebo-controlled trial. Penciclovir Cream Herpes Labialis Study Group. Clin Ther. 2000;22(1):76-90.

27. Pacifico A, Damiani G, Iacovelli P, et al. Photoadaptation to ultraviolet B TL01 in psoriatic patients. J Eur Acad Dermatol Venereol. 2020;34(8):1750-4.

28. Pacifico A, Damiani G, Iacovelli P, et al. NB-UVB plus oral polypodium leucotomos extract display higher efficacy than NB-UVB alone in patients with vitiligo. Dermatol Ther. 2021;34:e14776.

29. Pacifico A, Iacovelli P, Damiani G, et al. 'High dose' vs. 'medium dose' UVA1 phototherapy in Italian patients with severe atopic dermatitis. J Eur Acad Dermatol Venereol. 2019;33(4):718-24. 